\title{
Pneumoperitoneum in laparoscopy and preoperative antihypertensive agents: A retrospective study
}

\author{
HYUNJEE KIM
}

\begin{abstract}
Department of Anesthesiology and Pain Medicine, School of Medicine, Kyungpook National University, Daegu 41944, Republic of Korea
\end{abstract}

Received May 17, 2019; Accepted July 29, 2019

DOI: $10.3892 /$ etm.2019.8076

\begin{abstract}
The purpose of the current study was to investigate the effects of preoperative antihypertensive agents on blood pressure (BP) changes after intraperitoneal $\mathrm{CO}_{2}$ insufflation during laparoscopic surgery. The medical records of patients ( $\geq 50$ years old) undergoing laparoscopic colon surgery were retrospectively analyzed $(n=342)$. The patients were divided into four groups: normotensive group (group N), monotherapy with angiotensin receptor blockers (ARBs) (group A), monotherapy with calcium channel blockers (CCBs) (group C), and combination therapy with ARBs and CCBs (group AC). The systolic BP changes were investigated, and their correlations with preoperative pulse pressure were analyzed. The degree of increase from baseline BP to that after $\mathrm{CO}_{2}$ insufflation was significantly lower in groups $\mathrm{A}$ [difference vs. group $\mathrm{N}$, 17.8; 95\% confidence interval $(\mathrm{CI}), 7.8-27.8 ; \mathrm{P}=0.001)$ and AC (18.2; 95\% CI, 8.7-27.7; $\mathrm{P}<0.001]$ compared with groups $\mathrm{N}$ and $\mathrm{C}$. The incidence of intraoperative hypotension was not significantly different among the groups. In groups $\mathrm{A}$ and $\mathrm{AC}$, the preoperative pulse pressure showed a strong correlation ( $\mathrm{r}=0.73$ and 0.83 , respectively) with the increase in $\mathrm{BP}$ after $\mathrm{CO}_{2}$ insufflation. In conclusion, patients with hypertension who received ARBs before surgery showed a strong correlation between the preoperative pulse pressure and BP fluctuation immediately after $\mathrm{CO}_{2}$ insufflation. Small BP fluctuations are suggested to indicate the positive role of ARBs. Large randomized controlled trials are required to validate these results and assess the effect of each antihypertensive agent. This trial was registered (Trial registration: http://www. who.int/ictrp/network/cris2/en/; CRIS; KCT0002595) on the 23 November 2017.
\end{abstract}

Correspondence to: Hyunjee Kim, Department of Anesthesiology and Pain Medicine, School of Medicine, Kyungpook National University, 130 Dongdeok-ro, Jung-gu, Daegu 41944, Republic of Korea

E-mail:hj_kim@knu.ac.kr

Key words: angiotensin receptor antagonists, antihypertensive agents, hypertension, laparoscopy, pneumoperitoneum, preoperative care

\section{Introduction}

As the prevalence of hypertension has increased over the years, an improved understanding of the pharmacology of antihypertensive agents and the pathophysiology of hypertension has become indispensable in anesthesia practice. Hypertension is associated with different etiologies including genetic, physiological, and environmental factors (1). Patients need treatment with appropriate antihypertensive agents to achieve their target blood pressure (BP) that may be possible with monotherapy; some patients may require two or more agents with different mechanisms of action for successful outcome (2). The common drugs of choice are calcium channel blockers (CCBs), angiotensin receptor blockers (ARBs) or angiotensin converting enzyme inhibitors (ACEIs), and diuretics. Moreover, data on the decision to continue or discontinue treatment with ARBs or ACEIs before noncardiac surgery is inconclusive (3).

The pathophysiological changes following carbon dioxide $\left(\mathrm{CO}_{2}\right)$ pneumoperitoneum lead to notable hemodynamic responses, and the use of preoperative antihypertensive agents in patients with hypertension affects these responses. This study noted the effects of preoperative antihypertensive agents on hemodynamic responses after intraperitoneal $\mathrm{CO}_{2}$ insufflation during laparoscopic surgery. Upon reviewing patients' medical records, there were three major types of antihypertensive agents taken by patients undergoing laparoscopic colon surgery: Monotherapy with ARBs, monotherapy with CCBs, and combination therapy with ARBs and CCBs. To contribute to the management of anesthesia in patients with hypertension, a preliminary retrospective analysis was designed to evaluate the impact of antihypertensive agents on BP changes following intraperitoneal $\mathrm{CO}_{2}$ insufflation during laparoscopic colectomy. It was hypothesized that combination therapy with ARBs and CCBs would not cause severe fluctuations in BP following intraperitoneal $\mathrm{CO}_{2}$ insufflation. In addition, preoperative pulse pressure-an indicator reflecting the blood vessel stiffness (4) was investigated, and the relationship between pulse pressure and BP fluctuations after $\mathrm{CO}_{2}$ insufflation was analyzed. As the age of patients may be related to the type of antihypertensive agent selected (5-7), this factor was adjusted with other potential confounding variables in the final analysis. 


\section{Materials and methods}

The Institutional Review Board of Keimyung University Dongsan Hospital (DSMC 2017-08-001) approved this retrospective study and waived the need for informed consent. The study was registered at http://cris.nih.go.kr (KCT0002595). The author retrospectively identified patients who underwent laparoscopic colon surgery under general anesthesia between January 2014 and June 2017 via medical records. The inclusion criteria were as follows: Elective laparoscopic colon surgery; age $\geq 50$ years; systolic $\mathrm{BP}<140 \mathrm{mmHg}$ measured at daytime on the day before surgery and diastolic BP $<90 \mathrm{mmHg}(8)$; and American Society of Anesthesiologists physical status classifications I and II. For patients with hypertension, additional inclusion criteria were as follows: On hypertensive medication for at least 1 year following diagnosis; $\leq 10 \mathrm{mg} /$ day of the CCB amlodipine; equivalent to $\leq 80 \mathrm{mg}$ /day of the ARB telmisartan; and systolic BP $<140 \mathrm{mmHg}$ measured at daytime on the day before surgery and diastolic $\mathrm{BP}<90 \mathrm{mmHg}(8)$. The exclusion criteria were as follows: Use of and antihypertensive agent other than ARBs and CCBs; severe obesity (body mass index [BMI] $\geq 35 \mathrm{~kg} / \mathrm{m}^{2}$ ); diabetes; heart failure; severe kidney disease; and pregnancy or lactation.

The patients were divided into four groups according to medication status: Control group without hypertension (normotensive subjects, group $\mathrm{N}$ ); and groups of patients receiving monotherapy with ARBs (group A), monotherapy with CCBs (group C), and combination therapy with ARBs and CCBs (group AC). All patients with hypertension continued to take antihypertensive agents until the morning of surgery.

In all cases, anesthesia care was provided by a team of anesthesiologists, and a standardized general anesthesia protocol was followed for all patients. Standard monitoring included pulse oximetry, electrocardiography, noninvasive $\mathrm{BP}$, temperature assessment, and capnography. The bladder of the BP cuff encircled $\geq 80 \%$ of the patient's arm (8). Vital signs were recorded every $5 \mathrm{~min}$ or more frequently, if needed, during anesthesia and post-anesthesia care unit stay. General anesthesia was induced using intravenous propofol $2 \mathrm{mg} / \mathrm{kg}$ and sufentanil $0.2 \mathrm{mcg} / \mathrm{kg}$. Laryngoscopy and tracheal intubation were facilitated using intravenous rocuronium $0.6-0.9 \mathrm{mg} / \mathrm{kg}$. During surgery, sevoflurane concentration was titrated to maintain a bispectral index value between 45 and 60 , and ventilation was adjusted to maintain an end-tidal $\mathrm{CO}_{2}$ level between 35 and $40 \mathrm{mmHg}$. The patients were placed in the Trendelenburg position after induction of anesthesia. $\mathrm{CO}_{2}$ was insufflated into the peritoneal cavity at a pressure of $12-15 \mathrm{mmHg}$.

The baseline BP and baseline pulse pressure were defined as the average systolic BP and average pulse pressure, respectively, measured during the daytime at the hospital ward the day before surgery. $\mathrm{CO}_{2}$ insufflation $\mathrm{BP}$ was defined as the average systolic BP measured within 10 min after intraperitoneal $\mathrm{CO}_{2}$ insufflation; plateau $\mathrm{BP}$ was defined as the average systolic BP measured within 30-60 min after intraperitoneal $\mathrm{CO}_{2}$ insufflation. The primary outcome was the difference between baseline $\mathrm{BP}$ and $\mathrm{CO}_{2}$ insufflation $\mathrm{BP}$. The first secondary outcome was the difference between $\mathrm{CO}_{2}$ insufflation BP and plateau BP. The other secondary

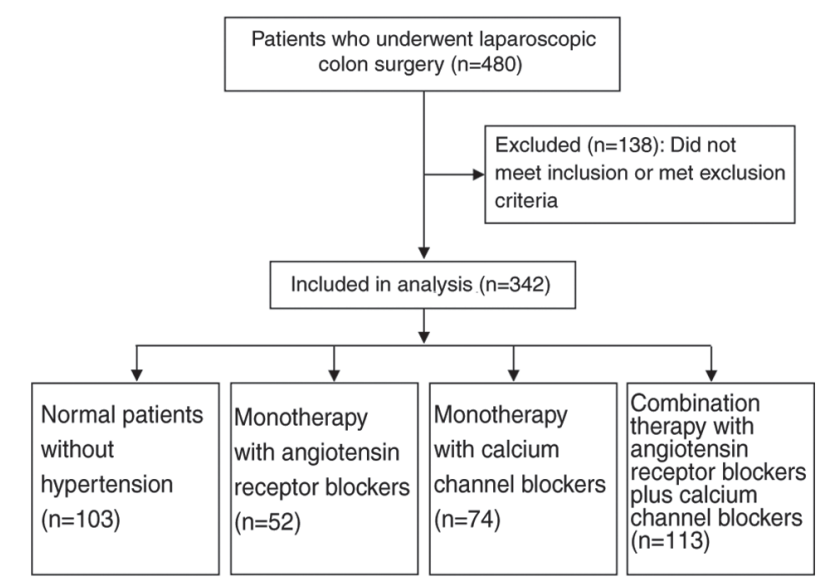

Figure 1. Patient flow diagram.

outcomes included incidence of intraoperative hypotension, use of vasoconstrictor agents, and correlation between the baseline pulse pressure and degree of BP change. Hypotension was defined as a $30 \%$ decline in systolic BP from baseline values (9), and the incidence of intraoperative hypotension was investigated.

Statistical analysis was performed using SPSS 24.0 (IBM Corp., Armonk, NY). Multiple comparison tests for different drug groups were conducted. Differences in BP were compared using analysis of covariance (ANCOVA) after adjusting for the effect of age, sex, height, BMI, and peak airway pressure. Logarithmic transformation of the differences in BP was performed to normalize the distributions. Categorical data in the cross-tabulation tables were compared using Pearson's chi-square or Fisher's exact test (Fisher's exact test was used in $2 \times 2$ contingency tables when the expected frequency was $<5$ in any cell). Differences in the incidence of hypotension among the groups were tested using the Mantel-Haenszel chi-square test to adjust for confounding factors. To analyze the correlation between pulse pressure and BP change, partial correlation coefficients were obtained after adjusting for confounding factors. $\mathrm{P}<0.05$ was considered to indicate a statistically significant difference.

\section{Results}

Among the 480 assessed patients, 138 were excluded (Fig. 1). The demographic data of the 342 remaining patients (Table I) showed no statistically significant differences among the groups with respect to sex, height, BMI, and peak inspiratory airway pressure. Only age was significantly different between group $\mathrm{N}$ and group $\mathrm{C}$, where patients in group $\mathrm{C}$ were older on average than those in group $\mathrm{N}$.

In the comparison between groups $\mathrm{N}$ and $\mathrm{A}$ and between groups $\mathrm{N}$ and $\mathrm{AC}$, there was a significant difference in the change from baseline to $\mathrm{CO}_{2}$ insufflation $\mathrm{BP}$, after adjusting for confounding factors (age, sex, height, BMI, and peak airway pressure); the degree of increase from baseline to $\mathrm{CO}_{2}$ insufflation BP was significantly lesser in groups $\mathrm{A}$ and $\mathrm{AC}$ (Tables II and III). The comparison between groups $\mathrm{C}$ and $\mathrm{A}$ and between groups $\mathrm{C}$ and $\mathrm{AC}$ also showed less increase in $\mathrm{BP}$ from baseline to $\mathrm{CO}_{2}$ insufflation BP in groups $\mathrm{A}$ and $\mathrm{AC}$. 
Table I. Demographic data, baseline BP and baseline pulse pressure.

\begin{tabular}{|c|c|c|c|c|}
\hline Characteristic & Group N (n=103) & Group A $(n=52)$ & Group C (n=74) & Group AC $(n=113)$ \\
\hline Age (years) & $65 \pm 9$ & $62 \pm 7$ & $71 \pm 6$ & $69 \pm 7$ \\
\hline \multicolumn{5}{|l|}{ Sex } \\
\hline Male & 40 & 21 & 40 & 65 \\
\hline Female & 63 & 31 & 34 & 48 \\
\hline Height (cm) & $160.6 \pm 9.8$ & $161.9 \pm 5.8$ & $161.2 \pm 8.2$ & $163.1 \pm 8.8$ \\
\hline $\operatorname{BMI}\left(\mathrm{kg} / \mathrm{m}^{2}\right)$ & $23.7 \pm 3.1$ & $24.8 \pm 2.3$ & $24.0 \pm 3.1$ & $23.3 \pm 3.0$ \\
\hline Baseline blood pressure (mmHg) & $122 \pm 14$ & $121 \pm 11$ & $124 \pm 13$ & $118 \pm 10$ \\
\hline Baseline pulse pressure (mmHg) & $48 \pm 10$ & $49 \pm 9$ & $52 \pm 13$ & $47 \pm 11$ \\
\hline Peak airway pressure (mmHg) & $23 \pm 2$ & $23 \pm 2$ & $23 \pm 2$ & $23 \pm 2$ \\
\hline
\end{tabular}

BP, blood pressure; BMI, body mass index.

Table II. Intraoperative changes in BP and incidence of hypotension.

\begin{tabular}{lcccc}
\hline Characteristic & Group N (n=103) & Group A (n=52) & Group C (n=74) & Group AC (n=113) \\
\hline $\mathrm{CO}_{2} \mathrm{BP}(\mathrm{mmHg})$ & $143 \pm 23$ & $125 \pm 20$ & $148 \pm 21$ & $125 \pm 17$ \\
Baseline BP-CO 2 BP & $-21 \pm 24$ & $-4 \pm 18$ & $-24 \pm 23$ & $-7 \pm 19$ \\
Plateau BP (mmHg) & $118 \pm 13$ & $103 \pm 8$ & $120 \pm 13$ & $101 \pm 10$ \\
$\mathrm{CO}_{2}$ BP-plateau BP & $26 \pm 19$ & $21 \pm 18$ & $29 \pm 18$ & $23 \pm 12$ \\
Incidence of hypotension & $43 / 60(43.7 \%)$ & $25 / 27(48.1 \%)$ & $39 / 35(52.7 \%)$ & $60 / 53(53.1 \%)$ \\
\hline
\end{tabular}

$\mathrm{BP}$, blood pressure; $\mathrm{CO}_{2} \mathrm{BP}$, average systolic blood pressure measured within 10 min after $\mathrm{CO}_{2}$ insufflation; Baseline $\mathrm{BP}-\mathrm{CO}_{2} \mathrm{BP}$, difference between baseline $\mathrm{BP}$ and $\mathrm{CO}_{2} \mathrm{BP}$; Plateau $\mathrm{BP}$, average systolic blood pressure measured within 30 min to $1 \mathrm{~h}$ after $\mathrm{CO}_{2}$ insufflation; $\mathrm{CO}_{2}$ BP-plateau BP, difference between $\mathrm{CO}_{2} \mathrm{BP}$ and plateau BP.

Table III. Comparison of BP difference and hypotension incidence.

\begin{tabular}{|c|c|c|c|c|c|c|}
\hline \multirow{2}{*}{ Comparison } & \multicolumn{2}{|c|}{ Baseline $\mathrm{BP}-\mathrm{CO}_{2} \mathrm{BP}$} & \multicolumn{2}{|c|}{$\mathrm{CO}_{2} \mathrm{BP}$-plateau BP } & \multicolumn{2}{|c|}{ Incidence of hypotension } \\
\hline & $\mathrm{P}$-value & Difference $(95 \% \mathrm{CI})$ & P-value & Difference $(95 \% \mathrm{CI})$ & P-value & OR $(95 \% \mathrm{CI})$ \\
\hline Group N vs. A & 0.001 & $17.8(7.8-27.8)$ & 0.26 & $5.0(-3.8-13.7)$ & 0.75 & $1.2(0.5-3.0)$ \\
\hline Group N vs. C & 0.45 & $4.1(-6.6-14.8)$ & 0.62 & $2.1(-6.3-10.4)$ & 0.66 & $0.8(0.3-2.1)$ \\
\hline Group N vs. AC & $<0.001$ & $18.2(8.7-27.7)$ & 0.13 & $5.2(-1.6-12.0)$ & 0.61 & $0.7(0.3-1.9)$ \\
\hline Group A vs. C & $<0.001$ & $21.9(10.3-33.5)$ & 0.28 & $5.5(-4.5-15.4)$ & 0.44 & $0.6(0.3-1.8)$ \\
\hline Group A vs. AC & 0.25 & $5.5(-3.8-14.7)$ & 0.51 & $2.5(-5.1-10.1)$ & 0.39 & $0.6(0.3-1.7)$ \\
\hline Group C vs. AC & 0.001 & $17.0(7.0-27.0)$ & 0.25 & $4.0(-2.9-10.9)$ & 0.79 & $0.8(0.4-2.2)$ \\
\hline
\end{tabular}

$\mathrm{BP}$, blood pressure; $\mathrm{CI}$, confidence interval; $\mathrm{OR}$, odds ratio; $\mathrm{CO}_{2} \mathrm{BP}$, average systolic blood pressure measured within 10 min after $\mathrm{CO}_{2}$ insufflation; Baseline $\mathrm{BP}-\mathrm{CO}_{2} \mathrm{BP}$, difference between baseline $\mathrm{BP}$ and $\mathrm{CO}_{2} \mathrm{BP}$; Plateau $\mathrm{BP}$, average systolic blood pressure measured within 30 min to $1 \mathrm{~h}$ after $\mathrm{CO}_{2}$ insufflation; $\mathrm{CO}_{2} \mathrm{BP}$-plateau $\mathrm{BP}$, difference between $\mathrm{CO}_{2} \mathrm{BP}$ and plateau BP.

The adjusted confounding factors included age, sex, height, BMI, and peak inspiratory airway pressure. The ANCOVA showed that only patient age had a significant effect on the difference in BP. Other comparisons showed no significant changes in BP. The incidence of intraoperative hypotension was not significantly different among the groups after adjusting for confounding factors (Table III).
There was a correlation between baseline pulse pressure and $\mathrm{BP}$ increase after $\mathrm{CO}_{2}$ insufflation in all patients, after adjusting for confounding factors (age, sex, height, BMI, and peak airway pressure) (Table IV). This correlation ranged from moderate to strong in patients with hypertension. Groups A and $\mathrm{AC}$ showed a stronger correlation than group C. A partial regression plot (adjusted for age, sex, height, BMI, and peak 
Table IV. Correlation between pulse pressure and BP change.

\begin{tabular}{|c|c|c|c|c|}
\hline Characteristic & Group N (n=103) & Group A $(n=52)$ & Group C (n=74) & Group AC $(n=113)$ \\
\hline \multicolumn{5}{|c|}{ Baseline $\mathrm{BP}-\mathrm{CO}_{2} \mathrm{BP}$} \\
\hline PCC & 0.31 & 0.73 & 0.54 & 0.83 \\
\hline P-value & 0.59 & $<0.001$ & 0.001 & $<0.001$ \\
\hline \multicolumn{5}{|c|}{$\mathrm{CO}_{2}$ BP-plateau BP } \\
\hline PCC & 0.22 & -0.64 & -0.44 & -0.30 \\
\hline P-value & 0.19 & 0.11 & 0.06 & 0.06 \\
\hline
\end{tabular}

$\mathrm{BP}$, blood pressure; $\mathrm{CO}_{2} \mathrm{BP}$, average systolic blood pressure measured within 10 min after $\mathrm{CO}_{2}$ insufflation; $\mathrm{Baseline} \mathrm{BP}-\mathrm{CO}_{2} \mathrm{BP}$, difference between baseline $\mathrm{BP}$ and $\mathrm{CO}_{2} \mathrm{BP}$; Plateau $\mathrm{BP}$, average systolic blood pressure measured within 30 min to $1 \mathrm{~h}$ after $\mathrm{CO}_{2}$ insufflation; $\mathrm{CO}_{2}$ BP-plateau BP, difference between $\mathrm{CO}_{2}$ BP and plateau BP; PCC, partial correlation coefficient.
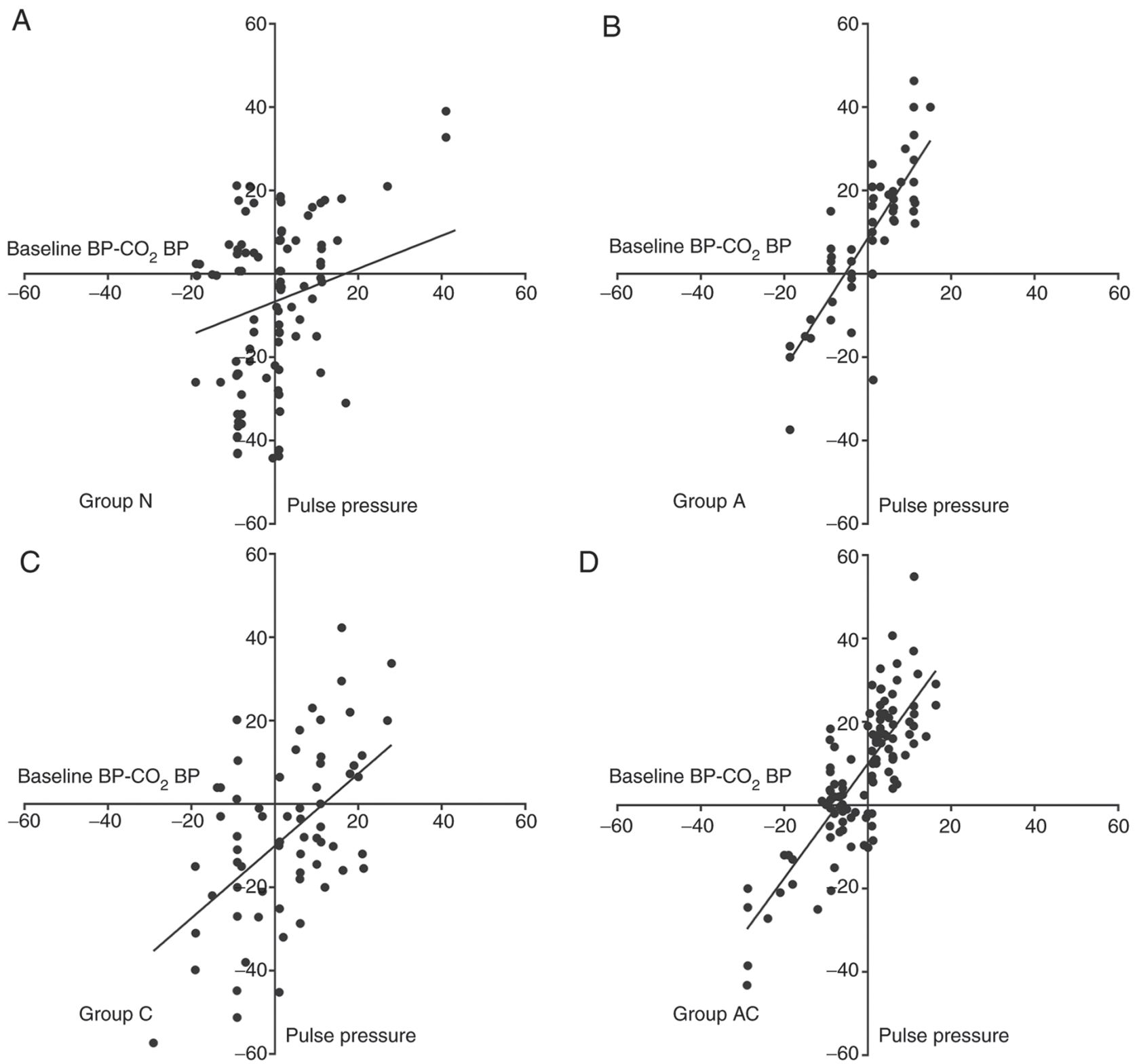

Figure 2. Partial regression plot. Partial regression plot (adjusted for age, sex, height, BMI, and peak airway pressure) of the residuals of baseline pulse pressure and the residuals of BP change after intraperitoneal $\mathrm{CO}_{2}$ insufflation demonstrates positive linear correlation. The patients were divided into four groups according to the medication status: (A) Control group without hypertension (normotensive subjects, group N) and (B) groups of patients receiving monotherapy with ARBs (group A), (C) monotherapy with CCBs (group C), and (D) combination therapy with ARBs and CCBs (group AC). BP, blood pressure; baseline BP, average systolic BP measured at the hospital ward the day before surgery; $\mathrm{CO}_{2} \mathrm{BP}$, average systolic $\mathrm{BP}$ measured within 10 min after $\mathrm{CO}$ insufflation; baseline $\mathrm{BP}-\mathrm{CO}_{2} \mathrm{BP}$, difference between baseline $\mathrm{BP}$ and $\mathrm{CO}_{2} \mathrm{BP}$; BMI, body mass index; $\mathrm{ARB}$, angiotensin receptor blockers; $\mathrm{CCB}$, calcium channel blockers. 
airway pressure) of baseline pulse pressure vs. BP increase after $\mathrm{CO}_{2}$ insufflation is shown in Fig. 2.

\section{Discussion}

In the present study, continuous administration of ARBs prior to surgery resulted in significantly less increase in BP after intraperitoneal $\mathrm{CO}_{2}$ insufflation. The correlation between the preoperative pulse pressure and increase in BP after intraperitoneal $\mathrm{CO}_{2}$ insufflation was positive and much stronger upon administration of ARBs compared to monotherapy with CCBs.

The current perioperative guidelines of the American College of Cardiology/American Heart Association suggest continuing the administration of antihypertensive agents prior to noncardiac surgery (10). Sudden withdrawal of antihypertensive medication can be associated with significant rebound hypertension $(10,11)$. Such uncontrolled hypertension is associated with many complications including myocardial ischemia, renal failure, and neurologic complications $(11,12)$. These concepts illustrate the benefits of using antihypertensive agents prior to surgery.

In the present study, the reason for the significant decrease in BP fluctuation with administration of ARBs is likely attributed to the relationship between the antihypertensive mechanism of the drugs and the mechanism of BP change during pneumoperitoneum. During pneumoperitoneum in laparoscopic surgery, increased catecholamine production, plasma renin activity, antidiuretic hormone production, and systemic vascular resistance lead to elevated BP (13). This is in direct contrast to the mechanism by which ARBs lower BP (14); thus, it can be inferred that ARBs effectively offset these processes.

Arterial stiffness is one of the major determinants of increased systolic BP and pulse pressure, especially in patients aged over 50 years $(4,15)$. Considering that our patients were older than 50 years, a strong correlation between the pulse pressure and BP increase during pneumoperitoneum in patients with hypertension suggests that the severity of arterial stiffness had a significant effect on BP fluctuation. Interestingly, when compared to $\mathrm{CCB}$ monotherapy, $\mathrm{ARB}$ administration showed a stronger correlation between pulse pressure and BP fluctuation, while the degree of BP fluctuation was much less. It can be interpreted that the groups that received ARBs had more severe arterial stiffness or that ARBs could better control hypertensive factors, other than arterial stiffness, during intraperitoneal $\mathrm{CO}_{2}$ insufflation.

The retrospective nature of this study led to many confounding variables. As preoperative factors, BP on the day before surgery was considered to be an inclusion criterion; however, the information on the degree of BP control before admission could not be obtained. Furthermore, the basis for prescribing each antihypertensive agent in each patient could not be investigated. As intraoperative factors, first, the depth of anesthesia and concentration of anesthetic agents were not controlled. Because intraperitoneal $\mathrm{CO}_{2}$ insufflation was performed within $15 \mathrm{~min}$ of anesthesia induction, the doses of intravenous and inhalational anesthetic agents used immediately after anesthesia induction were likely to be similar in terms of depth of anesthesia and analgesia. Second, the variables influencing BP and intraperitoneal pressure were not adjusted. All patients were placed in the Trendelenburg position, but the angles were not identical. Intraperitoneal $\mathrm{CO}_{2}$ pressure changed frequently depending on the surgical field. The difference in the intervention according to the lesion and mesenteric stimulation could lead to autonomic nervous system reflexes and BP change. To adjust factors affecting intraoperative pressure, the $\mathrm{BP}$ at the beginning of $\mathrm{CO}_{2}$ insufflation was compared with the mean BP within 30-60 min after insufflation. This is supported by the knowledge that hemodynamic changes begin to return to the pre-insufflation state via compensatory mechanisms approximately $15 \mathrm{~min}$ after gas insufflation (16). Nonetheless, the potential impact of each of these factors remains a limitation of this study. Third, as all hemodynamic elements were not analyzed and the entire period of pneumoperitoneum was not compared, the interpretation of results may not be accurate. Fourth, the use of vasoactive agents may have affected this outcome. Despite these uncontrolled confounding factors, the results of this study of $\mathrm{BP}$ changes after intraperitoneal $\mathrm{CO}_{2}$ insufflation under ARB administration may provide clues to further studies on antihypertensive agents.

In this study, compared to patients without hypertension, those with hypertension who were continuously administered ARBs before surgery had significantly less change in BP after intraperitoneal $\mathrm{CO}_{2}$ insufflation and no significant hypotension during surgery. Therapy with ARBs yielded a stronger correlation between the preoperative pulse pressure and $\mathrm{BP}$ increase following pneumoperitoneum than that with $\mathrm{CCB}$ monotherapy; moreover, the degree of BP fluctuation was also much less with ARB therapy. This suggests that ARBs as preoperative antihypertensive agents may play a positive role in managing BP in laparoscopic surgery. However, larger controlled trials of precise hemodynamic monitoring in consideration of potential confounding factors are needed to validate these results. The preliminary data on the effects of antihypertensive agents on BP during laparoscopic surgery presented herein lay the foundation for future randomized controlled trials and are expected to contribute to optimal preoperative and intraoperative management.

\section{Acknowledgements}

Not applicable.

\section{Funding}

No funding was received.

\section{Availability of data and materials}

The datasets used and/or analyzed during the present study are available from the corresponding author on reasonable request.

\section{Authors' contributions}

HK designed the study, obtained the data, analyzed and interpreted the data, and drafted and critically revised the manuscript.

\section{Ethics approval and consent to participate}

The study was approved by the Ethics Committee of Keimyung University Dongsan Hospital (Daegu, South Korea), with a waiver for the need for informed consent. 


\section{Patient consent for publication}

Not applicable.

\section{Competing interests}

The author declares that they have no competing interests.

\section{References}

1. Kakar P and Lip GY: Towards understanding the aetiology and pathophysiology of human hypertension: Where are we now? J Hum Hypertens 20: 833-836, 2006.

2. Gradman AH, Basile JN, Carter BL, Bakris GL, Materson BJ, Black HR, Izzo JL Jr, Oparil S and Weber MA: Combination therapy in hypertension. J Am Soc Hypertens 4: 90-98, 2010.

3. London MJ:Preoperative administration of angiotensin-converting Enzyme inhibitors or Angiotensin II receptor blockers: Do we have enough 'VISION' to stop it? Anesthesiology 126: 1-3, 2017.

4. Safar ME, Levy BI and Struijker-Boudier H: Current perspectives on arterial stiffness and pulse pressure in hypertension and cardiovascular diseases. Circulation 107: 2864-2869, 2003.

5. James PA, Oparil S, Carter BL, Cushman WC, Dennison-Himmelfarb C, Handler J, Lackland DT, LeFevre ML, MacKenzie TD, Ogedegbe O, et al: 2014 evidence-based guideline for the management of high blood pressure in adults: Report from the panel members appointed to the Eighth Joint National Committee (JNC 8). JAMA 311: 507-520, 2014.

6. Mancia G,Fagard R, Narkiewicz K, Redón J,Zanchetti A, Böhm M, Christiaens T, Cifkova R, De Backer G, Dominiczak A, et al: 2013 ESH/ESC Guidelines for the management of arterial hypertension: The Task Force for the management of arterial hypertension of the European Society of Hypertension (ESH) and of the European Society of Cardiology (ESC). J Hypertens 31: 1281-1357, 2013.

7. Sever P: New hypertension guidelines from the National Institute for Health and Clinical Excellence and the british hypertension society. J Renin Angiotensin Aldosterone Syst 7: 61-63, 2006.
8. Whelton PK, Carey RM, Aronow WS, Casey DE Jr, Collins KJ, Dennison Himmelfarb C, DePalma SM, Gidding S, Jamerson KA Jones DW, et al: 2017 ACC/AHA/AAPA/ABC/ACPM/AGS/ $\mathrm{APhA} / \mathrm{ASH} / \mathrm{ASPC} / \mathrm{NMA} / \mathrm{PCNA}$ Guideline for the prevention, detection, evaluation, and management of high blood pressure in adults: A report of the American College of Cardiology/American Heart Association Task Force on Clinical Practice Guidelines. Hypertension 71: 1269-1324, 2018.

9. Meersschaert K, Brun L, Gourdin M, Mouren S, Bertrand M, Riou B and Coriat P: Terlipressin-ephedrine versus ephedrine to treat hypotension at the induction of anesthesia in patients chronically treated with angiotensin converting-enzyme inhibitors: A prospective, randomized, double-blinded, crossover study. Anesth Analg 94: 835-840, 2002.

10. Fleisher LA, Fleischmann KE, Auerbach AD, Barnason SA, BeckmanJA,BozkurtB,Davila-Roman VG,Gerhard-Herman MD, Holly TA, Kane GC, et al: 2014 ACC/AHA guideline on perioperative cardiovascular evaluation and management of patients undergoing noncardiac surgery: A report of the American College of Cardiology/American Heart Association Task Force on Practice Guidelines. Circulation 130: 2215-2245, 2014.

11. Piccoli GB, Anania P, Biancone L, Mezza E, Vischi M, Jeantet A, Rabbia C, Savio D, Rossatto D, Gai M, et al: Hypertensive rebound after angiotensin converting enzyme inhibitor withdrawal in diabetic patients with chronic renal failure. Nephrol Dial Transplant 16: 1084-1085, 2001.

12. Malaty $\mathbf{J}$ and Malaty IA: Hypertensive urgency: An important aetiology of rebound hypertension. BMJ Case Rep 2014: pii: bcr2014206022, 2014.

13. Gerges FJ, Kanazi GE and Jabbour-Khoury SI: Anesthesia for laparoscopy: A review. J Clin Anesth 18: 67-78, 2006.

14. Burnier M and Brunner $\mathrm{H}$ : Angiotensin II receptor antagonists. Lancet 355: 637-645, 2000.

15. Safar ME: Systolic blood pressure, pulse pressure and arterial stiffness as cardiovascular risk factors. Curr Opin Nephrol Hypertens 10: 257-261, 2001.

16. Zuckerman RS and Heneghan S: The duration of hemodynamic depression during laparoscopic cholecystectomy. Surg Endosc 16: 1233-1236, 2002. 